\title{
TUMORES ADENOMATOIDES UTERINOS: ESTUDIO ANATOMO-PATOLÓGICO E INMUNOHISTOQUÍMICO DE 32 CASOS.
}

\author{
Luis Contreras M. ${ }^{1}$, Luis Contreras S. ${ }^{a}$, Ingrid Plass D. ${ }^{a}$, Paolo Ricci A. ${ }^{2}$ \\ 1 Servicio de Anatomía Patológica, ${ }^{2}$ Departamento de Obstetricia y Ginecología, Clínica Las Condes.
}

a Alumnos, Facultad de Medicina, Universidad Andrés Bello.

\section{RESUMEN}

Antecedentes: Los tumores adenomatoides (TA) son poco frecuentes. Se encuentran principalmente en el aparato reproductor femenino y especialmente en el útero. No existen reportes a nivel nacional sobre estos. Objetivos: Describir las características histológicas e inmunohistoquímicas de los TA uterinos. Método: Estudio descriptivo de 32 muestras ingresadas al Servicio de Anatomía Patológica de Clínica Las Condes. Las muestras estudiadas fueron recopiladas entre noviembre de 1999 y noviembre de 2008. Resultados: El diagnóstico de TA fue realizado en 21 histerectomías y 11 miomectomías. En 14 casos se diagnosticaron como lesiones nodulares únicas $(43,8 \%$ ) y en 18 junto a leiomiomas $(56,2 \%)$. El tamaño promedio de las lesiones únicas fue $2,6 \mathrm{~cm}$, significativamente mayor que aquellas asociadas a leiomiomas. El patrón histológico predominante más frecuente correspondió al tipo angiomatoide $(81,3 \%)$, seguido por los patrones adenoide $(9,4 \%)$, sólido $(6,3 \%)$ y mixto $(3 \%)$, no se encontraron TA quísticos. El estudio inmunohistoquímico fue positivo en el $100 \%$ de los casos para citoqueratina AE1/AE3, calrretinina, vimentina y D2-40. Fue negativo para CD31 y CEA. Sólo un 6,3\% (2 casos) fue positivo para citoqueratina 5/6. Conclusiones: Los TA corresponden a tumores benignos de origen mesotelial. Generalmente su diagnóstico es un hallazgo. Al presentarse en el útero, se confunden generalmente con leiomiomas o se presentan en conjunto con estos. En base a lo anterior el tratamiento de estas lesiones debe ser conservador, bastando con la resección del tumor.

\section{PALABRAS CLAVE: Tumor adenomatoide uterino, tumor benigno uterino, inmunohistoquímica, tumor mesotelial}

\section{SUMMARY}

Background: The adenomatoid tumors (AT) are rare. They are found mainly in the female reproductive system and especially in the uterus. There is not national reporting on these. Objective: To describe the histological and immunohistochemical features of uterine AT. Method: Descriptive study of 32 patients admitted to the Pathology Department of Clínica Las Condes. The cases studied were collected between November 1999 and November 2008. Results: The diagnosis of AT was performed in 21 hysterectomies and 11 myomectomies. In 14 patients were diagnosed as nodular single lesions (43.8\%) and in 18 cases associated with leiomyomas $(56.2 \%)$, the average size of single lesions was $2.6 \mathrm{~cm}$, significantly greater than those associated with leiomyomas. The predominant histologic type most often correspond to angiomatoid (81.3\%), followed by adenoid patterns $(9.4 \%)$, solid $(6.3 \%)$ and mixed $(3 \%)$, the cystic pattern was not observed. The 
immunohistochemical study of ST was positive in $100 \%$ for cytokeratin AE1/AE3, calrretinin, vimentin and D2-40. It was negative for CD31 and CEA. Only 6.3\% (2 cases) were positive for cytokeratin $5 / 6$. Conclusio$n s$ : The AT is a benign tumor of mesothelial origin. Usually the diagnosis is a finding. In the uterus they are generally mistaken with leiomyomas or it is in associated with them. Based on the foregoing the treatment of AT should be conservative, only with resection.

\section{KEY WORDS: Uterine adenomatoid tumor, benign uterine tumor, immunohistochemical, mesothelial tumor}

\section{INTRODUCCIÓN}

Los tumores adenomatoides (TA) son lesiones neoplásicas benignas, poco frecuentes, de origen mesotelial. Este término fue utilizado por primera vez en 1945 por Golden y Ash (1). Se trata de tumores que suelen ser únicos, afectando mayoritariamente al aparato reproductor femenino, y principalmente al útero durante la edad reproductiva (2-4). También pueden encontrarse en el aparato genital masculino (5), glándula suprarrenal, linfonodos, mediastino, corazón, páncreas y pleura (6-9).

El origen mesotelial de esta neoplasia se basa en estudios inmunohistoquímicos y reportes ultraestructurales que confirman esta teoría $(10,11)$. Estos tumores generalmente se encuentran de manera incidental en histerectomías realizadas por otras causas, habitualmente leiomiomas (12). Sin embargo, dependiendo de su localización, pueden causar sintomatología ginecológica, como dolor e infertilidad $(13,14)$.

Macroscópicamente se presentan como formaciones nodulares bien delimitadas, de aspecto arre- molinado, consistencia firme y de tamaño variable, habitualmente asociados a leiomiomas uterinos $(14,15)$.

Histológicamente se describen cuatro patrones: angiomatoide, adenoide, sólido y quístico $(16,17)$. El angiomatoide se caracteriza por formación de espacios pseudovasculares revestidos por células planas o cúbicas bajas, sin elementos sanguíneos en sus lúmenes, y con descamación luminal aislada de células de aspecto mesotelial (Figura 1A). El adenoide se caracteriza por un aspecto glandular con células cúbicas altas (Figura 1B). El sólido está formado por cordones celulares epitelioides sin patrón especial (Figura 1C). El quístico es de ubicación habitualmente subseroso, como su nombre lo indica está constituido por formación quística uni o multilocular revestida por células cúbicas o planas de origen mesotelial.

Con el objetivo de describir las características histopatológicas e inmunohistoquímicas de los TA y su diagnóstico diferencial, presentamos y analizamos una serie de 32 casos.
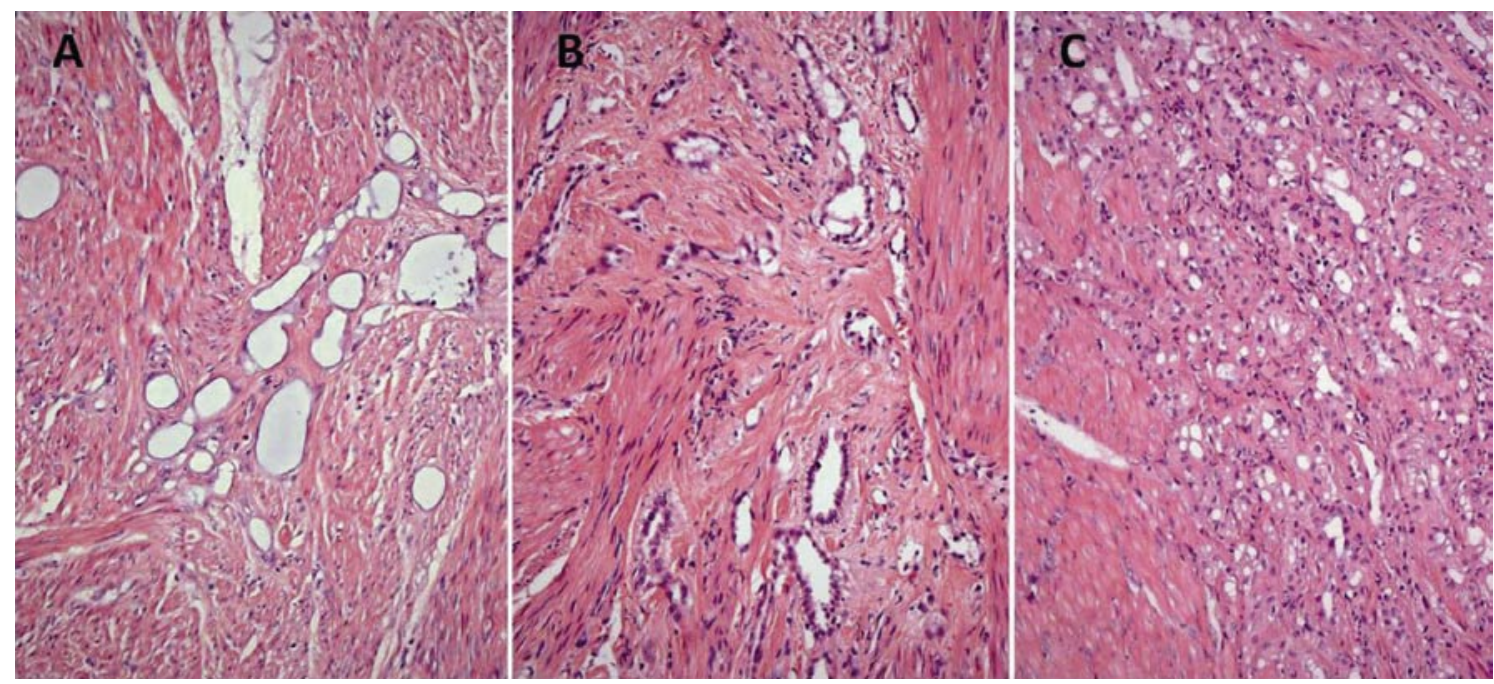

Figura 1. Patrones histológicos en tumores adenomatoides uterinos. Tinción con Hematoxilina-Eosina, observación con aumento 20X. A: angiomatoide; B: adenoide; C: sólido. 


\section{PACIENTES Y MÉTODO}

Se analizaron en forma retrospectiva y descriptiva 32 casos incluidos en los archivos del Servicio de Anatomía Patológica de Clínica Las Condes, entre noviembre de 1999 y noviembre de 2008 . En todos los casos el diagnóstico de TA se había realizado a través del aspecto morfológico de las muestras y/o estudio inmunohistoquímico.

El promedio de edad de las pacientes fue de 43 años (rango: 29 a 57 años) con una mediana de 44 años. Se revisaron los antecedentes clínicos de cada paciente.

Con respecto a las muestras, el análisis macroscópico incluyó características como el tamaño de las lesiones, su localización, la superficie de corte y color. Se revisaron las láminas histológicas y se determinó el patrón característico correspondiente a cada caso. Para el estudio inmunohistoquímico se seleccionaron áreas representativas, confeccionándose microarreglos tisulares con el manual TMA kit (3D HISTECH), cuando la cantidad de tejido remanente en las inclusiones de parafina así lo permitió.

Para realizar el diagnostico inmunohistoquímico de lesiones de origen mesotelial, se requiere demostrar la presencia de al menos 2 o 3 de los siguientes anticuerpos específicos, entre otros: citoqueratina $A E 1 / A E 3$, citoqueratina $5 / 6$, calrretinina, D2-40 y vimentina. Además, este diagnóstico exige que se encuentren negativos para el antígeno carcinoembrionario (CEA) y CD31 (18). Adicionalmente se evaluó la actividad proliferativa de la lesión con el uso del antígeno de proliferación Ki-67.

\section{RESULTADOS}

El diagnóstico de TA fue realizado en 21 piezas obtenidas a través de histerectomía $(65,6 \%)$, de las cuales 13 incluyeron uno o ambos anexos. En las 11 pacientes restantes, el diagnóstico se realizó en muestras obtenidas a través de supuestas miomectomías $(34,7 \%)$. El diagnóstico clínico presuntivo, en la solicitud de biopsia, fue mayoritariamente de miomas $(68,8 \%)$, seguido de miomas más adenomiosis (12,5\%) (Tabla I). En 14 pacientes el tumor adenomatoide fue diagnosticado como lesión única nodular $(43,8 \%)$ y en 18 casos junto a leiomiomas $(56,2 \%)$.

Las lesiones se ubicaron predominantemente a nivel subseroso en 14 casos (43.8\%), subseroso e intramural en 9 casos $(28,1 \%)$, intramural en 6 casos $(18,8 \%)$, y no fue posible evaluar su ubicación en 3 casos $(9,3 \%)$.

Las lesiones se presentaron como nodulares
Tabla I

DIAGNÓSTICO CLÍNICO EN LAS 32 PACIENTES

\begin{tabular}{lcc}
\hline Diagnóstico & $\mathrm{n}$ & $\%$ \\
\hline Miomas & 22 & 68,8 \\
Miomas y adenomiosis & 4 & 12,5 \\
Adenomiosis & 3 & 9,4 \\
Pólipo endometrial & 1 & 3,1 \\
Tumor ovárico & 1 & 3,1 \\
Prolapso uterino & 1 & 3,1 \\
Total & 32 & 100,0 \\
\hline
\end{tabular}

sólidas en 29 casos (91\%), nodular mixoides en 1 caso $(3 \%)$, en forma difusa en 1 caso $(3 \%)$ y no evaluable en 1 caso, correspondiente a una supuesta miomectomía (3\%).

En casos de TA como única lesión nodular, el tamaño promedio fue de $2,6 \mathrm{~cm}$ de diámetro (rango: 0,8 y $4,5 \mathrm{~cm}$ ), con una mediana de $2 \mathrm{~cm}$. En los casos de lesiones asociadas a leiomiomas, el tamaño promedio fue 1,2 cm (rango: 0,5 y $2 \mathrm{~cm}$ ), con una mediana de $1 \mathrm{~cm}$. La diferencia de tamaño entre los TA que se presentaron como lesión nodular única y los asociados a leiomiomas, fue significativa (T Student, $\mathrm{p}=0,004)$. El número promedio de leiomiomas en casos combinados con TA (18 casos) fue de 4,3 (rango: 1 y 10), con mediana de 3.

El patrón histológico más frecuente fue el angiomatoide $(81,3 \%)$, seguido del adenoide $(9,4 \%)$. El resto de los casos correspondió a asociación de patrones. No se encontraron como patrones únicos el tipo sólido ni quístico (Tabla II).

En el estudio histológico de piezas de histerectomía con o sin anexectomía, se encontraron otros hallazgos patológicos, entre los que se registró: pólipo endometrial adenomiomatoso en 1 caso, pólipo endometrial hiperplásico sin atipias en 1 caso, endometriosis tubo-ovárica en 4 casos y teratoma

\section{Tabla II \\ PATRÓN HISTOLÓGICO PREDOMINANTE EN LOS TUMORES ADENOMATOIDES}

\begin{tabular}{lcc}
\hline Tipo histológico & $\mathrm{n}$ & $\%$ \\
\hline Angiomatoide & 26 & 81,3 \\
Adenoide & 3 & 9,4 \\
Angiomatoide + Adenoide & 2 & 6,3 \\
Angiomatoide + Adenoide + Sólido & 1 & 3,0 \\
Total & 32 & 100,0 \\
\hline
\end{tabular}


quístico ovárico maduro en 1 caso. No se encontraron lesiones neoplásicas malignas.

El estudio inmunohistoquímico por TMA fue realizado en 21 casos. En los 11 casos restantes no fue posible, debido a lo escaso de la muestra remanente en las inclusiones de parafina. Sin embargo, al momento del diagnóstico en 3 de ellos se les realizó un examen inmunohistoquímico parcial, que fue suficiente para confirmar el diagnóstico con este método.

La distribución de tinciones inmunohistoquicas demostró positividad intensa difusa en todos los casos estudiados para calrretinina, D2-40, vimentina y citoqueratina AE1/AE3 (Figura 2). Solo 2 lesiones $(6,3 \%)$ fueron positivas para citoqueratina $5 / 6$, y ningún caso se tiñó con CD31 ni CEA (Tabla III)
(Figura 3). Se incluyó un caso de angioma uterino como control para tinción positiva de CD31 (Figura 3C). La tinción con antígeno de proliferación Ki-67 fue positiva en el $100 \%$ de los casos estudiados con índice inferior al $1 \%$ en todos ellos.

El seguimiento promedio de estas pacientes fue de 65 meses (rango: 10 a 118 meses), con mediana de 74 meses. No se han registrado recidivas hasta el cierre del seguimiento.

\section{DISCUSIÓN}

Aún cuando los TA son muy poco frecuentes, y la gran mayoría de los reportes en la literatura médica corresponden a casos aislados, o pequeñas series, llama la atención que no hay publicaciones

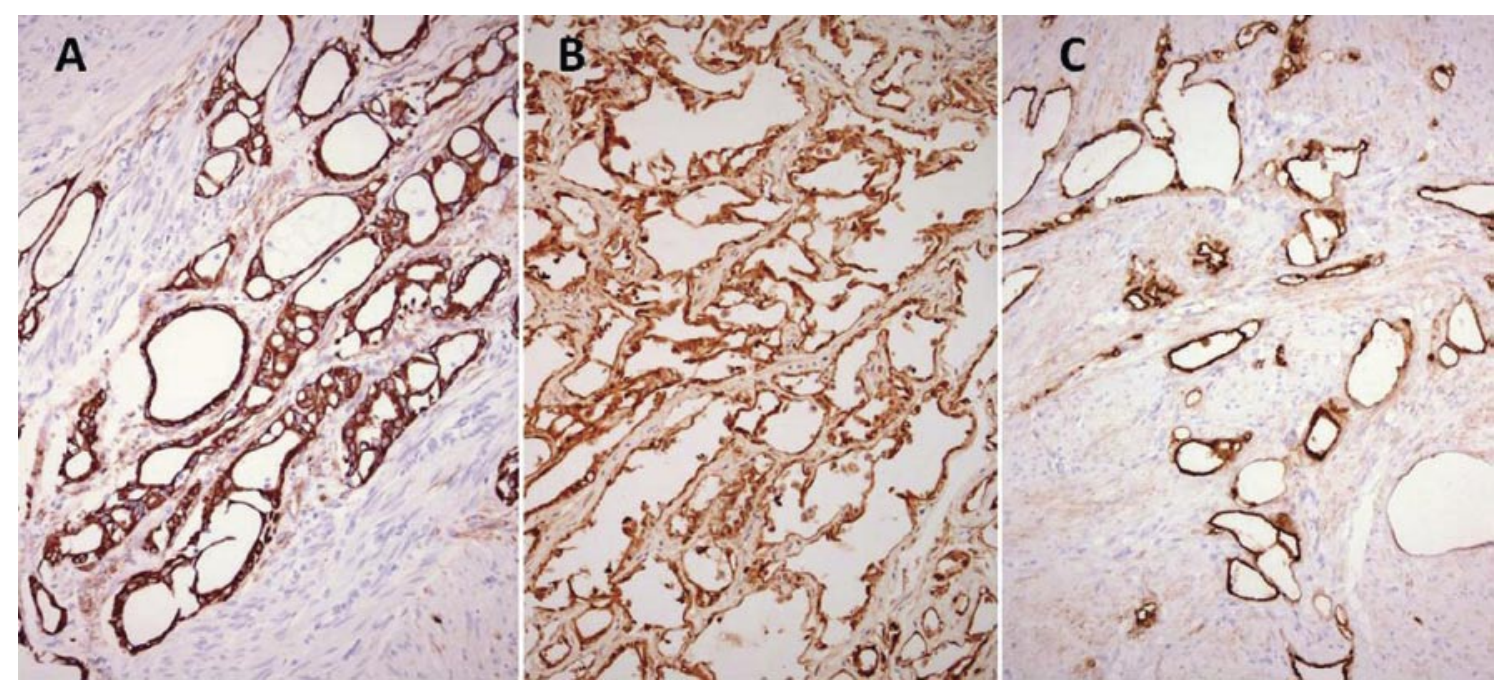

Figura 2. Tinción inmunohistoquímica positiva en tumor adenomatoide uterino. Observación con aumento de 20X. A: citoqueratina AE1/AE3; B: calrretinina; C: D2-40.

\section{Tabla III \\ RESULTADO DEL ESTUDIO INMUNOHISTOLÓGICO EN 21 CASOS}

\begin{tabular}{lcc}
\hline Anticuerpo & Casos positivos & \% casos positivos \\
\hline CEA , COL 1 & 0 & 0 \\
CK 5/6, CK5/6.007 & 2 & 6,3 \\
Calrretinina & 21 & 100 \\
D2-40 & 21 & 100 \\
Vimentina & 21 & 100 \\
Citoqueratina AE1/AE3 & 21 & 100 \\
CD31 & 0 & 0 \\
\hline
\end{tabular}

en nuestro medio. Por esta razón, nos interesó analizar una casuística que hemos recopilado a través de los años. Algunos estudios han determinado que los TA pueden diagnosticarse hasta en el $1 \%$ de las histerectomías (19). Por otro lado, debemos tener presente que se trata de la cirugía más realizada en ginecología y la más frecuente en mujeres, estimándose hasta 600.000 cada año en EEUU. De estas la causa mayor es por leiomiomas sintomáti$\cos (20)$. Por estos motivos, no es de extrañar que en la presente serie, en el $65,6 \%$ correspondieron a diagnósticos realizados en úteros obtenidos de histerectomías, con el diagnóstico más frecuente de leiomiomas, en la solicitud de estudio anatomopatológico. 


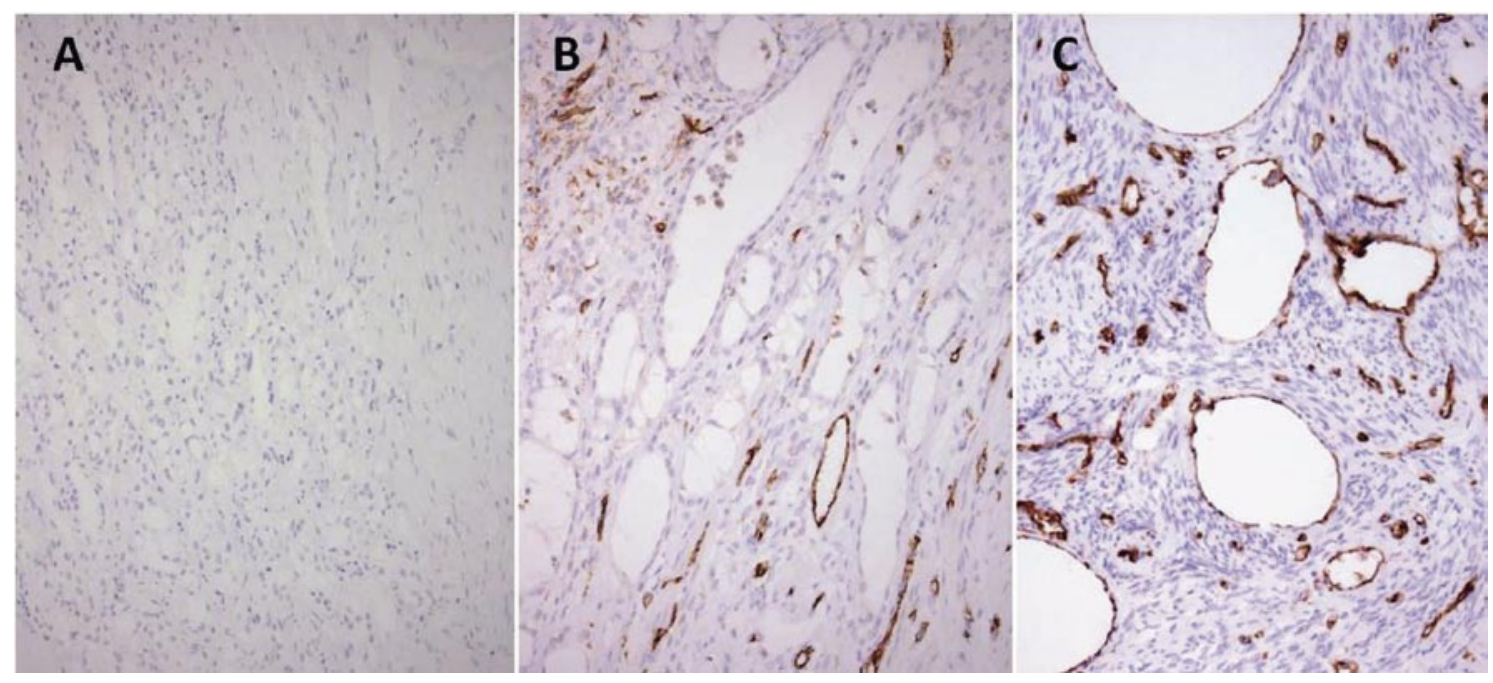

Figura 3. Tinción inmunohistoquímica negativa en tumor adenomatoide uterino y positiva en angioma de control. Observación con aumento de 20X. A: tinción inmunohistoquímica CEA negativa en tumor adenomatoide uterino. B: tinción inmunohistoquímica CD31 negativa en tumor adenomatoide uterino. C: tinción inmunohistoquímica CD31 positiva en angioma uterino.

Los TA generalmente son nodulares, sin embargo, se han descrito casos difusos que infiltran prácticamente todo el miometrio. En la mitad de los reportes que hay en la literatura, estos últimos corresponden a casos en pacientes inmunodeprimidos (2,21-23). Algunos autores creen que la variedad difusa podría corresponder a una entidad biológica distinta (24). En nuestra serie sólo 1 caso se presentó como difuso. Este patrón puede simular un adenocarcinoma (25). Menos frecuente aún, se han descrito algunos casos de presentación de TA en forma simultánea en diferentes órganos, como útero, ovario y apéndice o útero y ovario $(14,26)$.

El diagnóstico de los TA, generalmente no es sospechado, constituyéndose con alta frecuencia en un hallazgo operatorio, tal como fue en los 32 casos que analizamos (12). En un estudio de 60 pacientes, solamente 4 casos fueron sospechados antes de la cirugía (17). Los exámenes de imágenes pre-quirúrgicos generalmente no permiten distinguir a estos tumores de los leiomiomas y/o adenomiosis $(27,28)$. Por otro lado, frecuentemente se pueden encontrar junto a miomas $(66,2 \%$ para la serie) $(14,15)$. El término tumor leiomioadenomatoide, fue utilizado por primera vez por Epstein (29).

Los síntomas y signos de los TA pueden ser variados y dependerán de la localización dentro del útero o el órgano en el cual se presenten. En la mayoría de los casos se confunden con los de leiomiomas (30), y como en muchos casos se presentan asociados a estos, no se diferencian en el estudio pre-operatorio, ni en los signos o síntomas previos. Se han descrito casos de infarto de TA que han consultado por dolor abdominal (14). En estas pacientes, debido a la necrosis de los tejidos comprometidos, se puede confundir con tumores malignos (31).

El tratamiento de los TA es la simple escisión, ya que la recurrencia es rara. Sin embargo, durante el 2005 se publicó un caso de recurrencia $(32,33)$. En nuestra serie, con una mediana de seguimiento registrado de 74 meses, no se han detectado signos o síntomas que sugieran recidiva.

El diagnóstico diferencial en el útero, debe realizarse con adenocarcinoma ya sea endometrial o metastásico, el estudio histológico contempla actividad mitótica ausente o baja, ausencia de atipía nuclear y la presencia de discretas proyecciones citoplasmáticas (34).

En nuestra serie, al igual que la literatura internacional, el patrón histológico predominante más frecuente fue el tipo angiomatoide, seguido del adenoide y sólido $(4,35)$ (Figura 1$)$. La tinción inmunohistoquímica con CD31 fue negativa en todas las lesiones estudiadas, lo que permite realizar el diagnóstico diferencial con lesiones de origen vascular (Figura 3).

El estudio inmunohistoquímico permite argumentar el origen mesotelial de los TA $(10,18,36)$. Es así como las células tumorales fueron positivas en nuestra serie en todos los casos estudiados, para calrretinina, D2-40, citoqueratina AE1/AE3 y vimen- 
tina, al igual que otras publicaciones $(10,30,36,37)$ (Figura 2). En nuestra casuística el estudio para citoqueratina $5 / 6$, habitualmente asociada a proliferación y tumores mesoteliales (18), sólo fue positivo en dos casos (6,3\%). Esto fue diferente a otros estudios, en que la positividad para este anticuerpo ha sido descrita superior al $80 \%(18,35)$. Todos los casos fueron negativos para CD31 y antígeno carcinógeno embrionario, al igual que otras series menores (35) (Tabla III).

\section{CONCLUSIONES}

Los TA corresponden a una entidad benigna y poco frecuente. El estudio inmunohistoquímico indica que son tumores benignos de origen mesotelial. Generalmente su diagnóstico es un hallazgo al realizar el estudio anatomo-patológico de la pieza operatoria. Al presentarse en el útero, se confunden generalmente con leiomomas o se encuentran en conjunto con estos. El tratamiento de estas lesiones debe ser conservador, bastando con la resección del tumor.

AGRADECIMIENTOS: A la Tecnóloga Jefe del Servicio de Anatomía Patológica de Clínica las Condes, Sra. María Angélica Garmendia Flores, por la confección de los microarreglos tisulares, la alta calidad de las tinciones inmunohistoquímicas realizadas y su apoyo en este trabajo.

\section{BIBLIOGRAFÍA}

1. Golden A, Ash JE. Adenomatoid tumors of the genital tract. Am J Pathol 1945;21:63-79.

2. Acikalin MF, Tanir HM, Ozalp S, Dundar E, Ciftci E, Ozalp E. Diffuse uterine adenomatoid tumor in a patient with chronic hepatitis $\mathrm{C}$ virus infection. Int J Gynecol Cancer 2009;19(2):242-4.

3. Zalud R, Michal M. What is your diagnosis? Answer: adenomatoid tumor of the uterus. Cesk Patol 2004;40(1):11-34.

4. Tiltman A. Adenomatoid tumors of the uterus. Int $\mathrm{J}$ Gyncol Pathol 2002;21(3):305.

5. Williams SB, Han M, Jones R, Andrawis R. Adenomatoid tumor of the testes. Urology 2004;63(4):779-81.

6. Raaf HN, Grant LD, Santoscoy C, Levin HS, AbdulKarim FW. Adenomatoid tumor of the adrenal gland: a report of four new cases and a review of the literature. Mod Pathol 1996;9(11):1046-51.

7. Minato H, Nojima T, Kurose N, Kinoshita E. Adenomatoid tumor of the pleura. Pathol Int 2009;59(8):56771.

8. Val-Bernal JF, Fogols J, Gómez-Román JJ. Incidental localized (solitary) epithelial mesothelioma of the pericardium: case report and literature review. Cardiovasc Pathol 2002;11(3):181-5.

9. Sollami S, Ben Salah I, Chelif M, Hafsia G, Ben Rhouma S, Nouira Y, Horchani A. Intrascrotal adenomatoid tumors. Tunis Med 2008;86(6):626.
10. Zhu L, Li B. Clinical pathological analysis of adenomatoid tumor in uterus and ovaries. Zhonghua Binq Li Xue Za Zhi 2001;30(1):43-5.

11. $\mathrm{Yu}$ JR, Wanq JL. Adenomatoid tumors in the uterus: an immunohistochemical and ultrastructural study. Zhonghua Fu Chan Ke Za Zhi 1994;29(12):727-8.

12. Buzzi A, Pezzica E, Crescini C. An adenomatoid tumor of the uterus. Minerva Ginecol 1994;46(6):359-6.

13. Butterwegge M, Krech R, Ohlenroth $G$. The so-called adenomatoid tumor of the uterus: a case of evaluation of primary sterility. Geburtshilfe Frauenheilkd 1995;55(1):53-5.

14. Honq R, Choi DY, Choi SJ, Lim SC. Multicentric infarcted leiomyoadenomatoid tumor: a case report. Int J Clin Exp Pathol 2009;2(1):99-103.

15. Amre R. Constantino J, Lu S, Charney D. Pathologic quiz case: a 52-year-old woman with a uterine mass. Leiomyo-adenomatoid tumor of the uterus. Arch Pathol Lab Med 2005;129(3):e77-8.

16. Rappa F, Ternullo MP. Adenomatoid tumor. Pathologica 2006;98(2):164-6.

17. Nogales FF, Isaac MA, Hardisson D, Bosincu L, Palacios $\mathrm{J}$, Ordi $\mathrm{J}$, et al. Adenomatoid tumors of the uterus: an analysis of 60 cases. Int $\mathrm{J}$ Gynecol Pathol 2002;21(1):34-40.

18. Marchevsky AM. Application of immunohistochemistry to the diagnosis of malignant mesothelioma. Arch Pathol Lab Med 2008;132(3):397-401.

19. Mainquené C, Hugol D, Hofman P, Clement N, BoiseIle JC, Huguet C, Poitout P. Adenomatoid tumors of the uterus. Study of 5 cases with immunohistochemical and ultrastructural confirmation of the mesothelial origin. Arch Anat Cystol Pathol 1996;44(4):174-9.

20. Merrill RM. Hysterectomy surveillance in the United States, 1997 through 2005. Med Sci Monit 2008;14(1): CR24-31.

21. Chenq CL, Wee A. Diffuse uterine adenomatoid tumor in a immunosuppressed renal transplant recipient. Int J Gynecol Pathol 2003;22(2):198-201.

22. Luchs J, Diel J, Katz DS. Diffuse adenotaid tumor of the uterus. AJR Am J Roentgenol 2000;175(2):554-5.

23. Duval H, Rioux-Leclerq N, Bauville E, Al Jaradi $M$, Burtin. Multinodular-adenomatoid tumor of the uterus in a patient with a renal allograft. Ann Pathol 2008;28(4):308-10.

24. Di Stefano D, Faticanti Scucchi L, Covello R, Martinazzoli A, Meli C, Bosman C. Uterine diffuse adenomatoid tumor. Does it represent a different biological entity? Gynecol Obstet Invest 1998;46(1):68-72.

25. Cserni G, Kocsis L, Pusztai Z, Godó G. Endometrial adenocarcinoma with coexisting adenomatoid tumor of the uterus. Gynecol Oncol 2003;90(1):207-10.

26. Hanada S, Okumura Y, Kaida K. Multicentric adenomatoid tumors involving uterus, ovary, and appendix. J Obstet Gynaecol Res 2003;29(4):234-8.

27. Mitsumori $A$, Morimoto $M$, Matsubara $S$, Yamamoto M, Akamatsu N, Hiraki Y. MR appearance of adenomatoid tumors of the uterus. $\mathrm{J}$ Comput Assist Tomogr 2000;24(4):610-3.

28. Tamai K, Togashi K, Ito T, Morisawa N, Fujiwara $T$, Koyama T. MR imaging finding of a adenomyosis: correlation with histopathologic features and diagnostic 
pitfalls. Radiographics 2005;25(1):21-40.

29. Epstein JL. Urologic disorders: Differential diagnosis in Pathology. New York, NY: Igasku-Shoin,1992;1734.

30. Dietterle S, Hantschick M, Stosiek P. Adenomatoid tumor of the uterus--2 cases reports. Zentralbl Gynakol 1997;119(2):82-5.

31. Skinnider BF, Young RH. Infarcted adenomatoid tumor; a report of five cases of a facet of a benign neoplasm that may cause diagnostic difficulty. Am Surg Pathol 2004;28(1):77-83.

32. Klintorp S, Grinsted L, Franzmann MB. Adenomatoid tumor of the uterus. Eur J Obstet Gynecol Reprod Biol 1993;50(3):255-7.

33. Sieunarine K, Cowie AS, Bartlett JD, Lindsay I, Smith JR. A novel approach in the management of a recurrent adenomatoid tumor of the uterus utilizing a Strassman technique. Int J Gynecol Cancer 2005;15(4):671-5.

34. Fajardo-Bernal L, Acosta-Forero J, Ricaurte-Guerrero O. Tumor adenomatoide del tracto genital femenino. Informe de dos casos de cuerpo uterino y ovario, y revisión de la literatura. Rev Colomb Obstet Ginecol 2009;60(1):83-8.

35. Canedo-Patzi AM, León-Bojorge B, de Ortíz-Hidalgo C. Adenomatoid tumor of the genital tract. Clinical, pathological and immunohistochemical study in 9 cases. Gac Med Mex 2006;142(1):59-66.

36. Yo H, Mori O, Ohaki Y, Kawamura T, Asano G. Three cases of adenomatoid tumor of the uterus. Nippon Ika Daigaku Zasshi 1996;63(4):294-8.

37. Satoh Y, Hiraguchi M, Matuura T, Takasu K. Immunohistochemical evidence of the mesothelial histogenesis of a uterine adenomatoid tumor. J Obstet Gynaecol Res 1998;24(2):91-101. 